\title{
Soil properties control decomposition of soil organic carbon: results from data-assimilation analysis
}

Xia Xu ${ }^{1,2}$, Zheng Shi ${ }^{2}$ Dejun $\mathrm{Li}^{3}$, Ana Rey ${ }^{4}$, Honghua Ruan ${ }^{1}$, Joseph M. Craine ${ }^{5}$, Junyi Liang ${ }^{2}$, Jizhong Zhou ${ }^{2,6}$,

Yiqi Luo ${ }^{2}$

${ }^{1}$ College of Biology and Environment, Co-Innovation Center for Sustainable Forestry in Southern China,

Nanjing Forestry University, Nanjing, Jiangsu Province, 210037, China

${ }^{2}$ Department of Microbiology \& Plant Biology, University of Oklahoma, Norman, OK, 73019, USA

${ }^{3}$ China Institute of Subtropical Agriculture, Chinese Academy of Sciences, Changsha, Hunan, 410125, China

${ }^{4}$ Department of Biogeography and Global Change, National Natural Science Museum, CSIC, Madrid, Spain

${ }^{5}$ Division of Biology, Kansas State University, Manhattan, KS, 66506, USA

${ }^{6}$ Institute for Environmental Genomics, University of Oklahoma, Norman, OK, 73019, USA

Author for Correspondence: Xia Xu \& Yiqi Luo

251 Bessey Hall, Ames, IA, 50011

Tel.: + 1 515-203-9925

E-mail: xuxia.1982@yahoo.com; yluo@ou.edu

Key words: soil organic carbon, decomposition, carbon pools, soil properties, clay content, data-assimilation 


\begin{abstract}
Soil properties, such as clay content, are hypothesized to control decomposition of soil organic carbon (SOC). However, these hypotheses of soil property-C decomposition relationships have not been explicitly tested at large spatial scales. Here, we used a data-assimilation approach to evaluate the roles of soil properties and environmental factors in regulating decomposition of SOC. A three-pool (active, slow, and passive) C-cycling model was optimally fitted with 376 published laboratory incubation data from soils acquired from 73 sites with mean annual temperature ranging from -15 to $26^{\circ} \mathrm{C}$. Our results showed that soil physical and chemical properties regulated decomposition rates of the active and the slow $\mathrm{C}$ pools. Decomposition rates were lower for soils with high clay content, high field water holding capacity (WHC), and high C:N ratio. Multifactor regression and structural equation modeling (SEM) analyses showed that clay content was the most important variable in regulating decomposition of SOC. In contrast to the active and slow C pools, soil properties or environmental factors had little effect on the decomposition of the passive $C$ pool. Our results show inverse soil property-C decomposition relationships and quantitatively evaluate the essential roles of soil texture (clay content) in controlling decomposition of SOC at a large spatial scale. The results may help model development and projection of changes in terrestrial C sequestration in future.
\end{abstract}




\section{Introduction}

Decomposition of soil organic matter (SOM) plays an essential role in terrestrial carbon (C) cycling (Trumbore, 1997; Knoblauch et al., 2013; Wang et al., 2013). It facilitates the growth and life of the soil biota by providing energy from $\mathrm{C}$ compounds and nutrients in inorganic forms. Considerable effort has been aimed at understanding the variability of decomposition rate of soil organic C (SOC) among soil types and under different climatic conditions (e.g. Craine et al., 2010; Wang et al., 2013). The requirement for an accurate accounting of this spatial variability is central to an accurate prediction of the amount of $\mathrm{C}$ released through this process. So far, however, it is still not satisfactorily known how SOC decomposition rates vary at a large spatial scale and which factors control SOM decomposition.

SOM consists of a continuum of components ranging from labile compounds that mineralize rapidly during the first stage of decomposition to more recalcitrant residues that accumulate during advanced stages of decomposition (Elberling et al., 2013; Xu et al., 2010a). It is generally agreed that SOC contains at least three identifiable $\mathrm{C}$ pools: the active, the slow (intermediate), and the passive pools (Parton et al., 1987; Trumbore, 1997; Luo et al., 2003). Much of the work in understanding and modeling soil $\mathrm{C}$ is the study of relative decomposition rates for various pools and their controlling factors. Empirical studies, including fractionation of SOM, soil respiration, laboratory incubations, and isotopic studies, have identified factors that regulate decomposition of SOC as (a) climatic factors, e.g. temperature, and water content (Thomsen et al., 1999; Wang et al., 2013); (b) soil properties, e.g. clay content (Schimel et al., 1994; Balesdent et al., 2000; Mtambanengwe et al., 2004), water holding capacity (WHC, 
Schønning et al., 1999), C:Nitrogen (C:N) ratio (Enríquez et al., 1993; Schädel et al., 2013), specific surface area (SSA) of soil mineral particles (Saggar et al., 1996), etc.; and (c) geographical variables, e.g. latitude and altitude (Craine et al., 2010; Wang et al., 2013).

Among all the factors, the primary role of soil texture in driving SOC decomposition has been well established in mineral soils at the small scale (e.g. site level). Decomposition of SOC is generally lower in soils with high clay concentrations (e.g. Schimel et al., 1985; Spain, 1990; Schimel et al., 1994; Balesdent et al., 2000; Mtambanengwe et al., 2004) as soil minerals can protect SOM from enzymatic decomposition (Baldock \& Skjemstad, 2000; Six et al., 2002). Chemically, the strength of the interaction of the chemical structure of SOC with minerals and organic soil components defines the degree of protection of SOC (Baldock \& Skjemstad, 2000; Six et al., 2002). Physically, a factor of soil texture, specific mineral surface area, and soil mineralogy defines the degree of protection of SOC (Baldock \& Skjemstad, 2000; Six et al., 2002).

Empirical studies have undoubtedly improved our understanding of decomposition of SOC. These observations have also allowed for development and validation of models used to simulate SOM dynamics. The challenge for empirical studies lies in that no single, satisfactory method yet exits to separate SOC into components with intrinsic pool-specific decomposition rates (Trumbore, 1997). Given this difficulty, SOM models (e.g. CENTURY) have played an important role in simulating decomposition of SOC (e.g. Parton et al., 1987; McGill, 1996; Burke et al., 2003). The heterogeneity of SOC in most of these models is represented by conceptually defining multiple pools, typically three to five, with varying inherent decay rates, 
assuming first-order kinetics modified by climatic and edaphic reduction factors (Parton et al., 1987; McGill, 1996; Six et al., 2002; Luo et al., 2003). However, many SOM models are developed and tested using experimental results, often restricted to the ecosystem or site scale and difficult to extrapolate to the large spatial scales. With the increased use of models for regional and global applications, understanding the roles of these control variables played on SOC decomposition becomes crucial.

The relationship between decomposition of SOC and various potential controlling factors remain unresolved and have been addressed differently in leading climate-carbon models (Burke et al., 2003). The unknowns in this issue may result in predictions of feedbacks to climate change from soil C uncertain (Friedlingstein et al., 2014). In this study, we developed a three-pool SOM decomposition model with C transfers between pools (active, slow, and passive, Fig. S1) and applied the techniques of data assimilation (also known as deconvolution analysis) to estimate decomposition rates of the active, the slow, and the passive SOC pools with laboratory soil incubation data sets. Laboratory incubation of soil samples, where plant roots are excluded, allow observation of the $\mathrm{CO}_{2}$ evolved from decomposition alone (Craine et al., 2010; Wang et al., 2013). We aimed to examine the roles of different factors play in decomposition of SOC and identify the most important controlling factors at a large spatial scale.

\section{Methods}

Incubation datasets 
The incubation datasets used in this analysis were obtained from researchers (Rey et al., 2006; Rey \& Jarvis, 2008; Craine et al., 2010; Haddix et al., 2011; Wang et al., 2013) and ten datasets were extracted from literature (Kätterer et al., 1998; Neff \& Hooper, 2002; Dutta et al., 2006; Schaeffer et al., 2007; Stewart et al., 2009; Cusack et al., 2010; Lavoie et al., 2011; Lee et al., 2012; Elberling et al., 2013; Knoblauch et al., 2013). All the incubation studies have been published (Table S1). Environmental factors and soil properties of the datasets varied among sites (Table S1). We chose the datasets to be included in this analysis according to the following criteria: (1) $\mathrm{CO}_{2}$ production had to be measured over the course of the incubation study; (2) the incubation study had to last at least seven months or longer to allow estimation of the relative contributions of different SOC pools. The seven month was chosen because previous studies reported that labile $\mathrm{C}$ pool in soil could be almost completely decomposed around 210 d (Conant et al., 2008; Xu et al., 2010b); (3) initial C concentration of the soils had to be available, and (4) $\mathrm{CO}_{2}$ production rates at the beginning of the incubation had to be higher than those at the end of the incubation in order to fit a first-order decay model. In total, laboratory constant-temperature incubation of 376 soil samples from 73 sites was included in this study (Fig. 1). Details on the lab incubation techniques, monitoring of $\mathrm{CO}_{2}$ production rates can be found the corresponding literature.

\section{Model description}

SOC is composed of a continuum of materials with varying chemical complexity and its dynamics usually need to be represented by models with more than one C pool (Trumbore, 1997; Xu et al., 2012). In this study, a three-pool C decomposition model, consisting of fast, 
slow, and passive pools and C transfers between pools, was developed to separate total measured $\mathrm{CO}_{2}$ production from the incubation experiments into pool-specific decay rates using deconvolution analysis (Fig. S1, Liang et al., 2015). The dynamics of soil C pool follows a first-order differential equation as described in the CENTURY and the Terrestrial ECOsystem (TECO) models (Parton et al., 1987; Luo et al., 2003) and can be expressed by a matrix equation (Luo \& Weng, 2011) as (Eq. 1):

$\frac{d P(t)}{d t}=A K P(t)$

where $A$ and $K$ are matrices as follows:

$$
\begin{aligned}
& A=\begin{array}{rrr}
1 & f_{1,2} & f_{3,1} \\
f_{2,1} & 1 & 0 \\
f_{3,1} & f_{3,2} & 1
\end{array} \\
& K=\operatorname{diag}(k)=\left(\begin{array}{ccc}
k_{1} & 0 & 0 \\
0 & k_{2} & 0 \\
0 & 0 & k_{3}
\end{array}\right)
\end{aligned}
$$

$P(t)=\left(P_{1}(t) P_{2}(t) P_{3}(t)\right)^{\top}$ is a $3 \times 1$ vector used to describe soil $C$ pool sizes. Matrix $A$ represents $C$ transfers between individual $C$ pools. $f_{i, j}$ is $C$ transfer coefficient, representing the fraction of the $C$ entering ith (row) pool from $j$ th (column) pool. $K$ is a $3 \times 3$ diagonal matrix that denotes decay rates of $\mathrm{C}$ pools. The observed total $\mathrm{CO}_{2}$ efflux in the three-pool model is the sum of pool-specific decomposition rates derived from a fast, a slow, and a passive $\mathrm{C}$ pools.

\section{Data assimilation}

Bayesian probabilistic inversion (Eq. 2) was used to estimate parameters of the $C$ dynamics model in this study (Xu et al., 2006):

$$
P(\theta \mid Z) \propto P(Z \mid \theta) P(\theta)
$$


Where posterior probability density function (PDF) $P(Z \mid \theta)$ of model parameters $(\theta)$ can be obtained from prior knowledge of parameters represented by a prior probability distribution $P(\theta)$ and information in the datasets represented by a likelihood function $P(Z \mid \theta)$. The prior PDF was specified as the uniform distributions over specific parameter ranges that were estimated according to previous studies (Trumbore, 1997; Craine et al., 2010). Ranges were: 1 -50 for active pool, $0.1-3$ for slow pool, and $1 \times 10^{-4}-0.1$ for passive pool in $\mathrm{mg} \mathrm{g}^{-1} \mathrm{C} \mathrm{d}^{-1}$. The likelihood function $P(Z \mid \theta)$ was calculated with the assumption that errors between observed and modeled values were independent distributed according to Eq. 3:

$$
P(Z \mid \theta) \propto \exp \left\{-\frac{1}{2 \sigma^{2}} \sum_{t \in o b s\left(Z_{i}\right)}\left[Z_{i}(t)-X_{i}(t)\right]^{2}\right\}
$$

where $Z_{i}(t)$ and $X_{i}(t)$ are the observed and modelled cumulative decay rates, respectively, and $\sigma_{i}(t)$ is the standard deviation of measurements.

The probabilistic inversion was carried on using the Metropolis-Hastings $(\mathrm{M}-\mathrm{H})$ algorithm to construct posterior PDFs of parameters. The $\mathrm{M}-\mathrm{H}$ algorithm samples random variables in high-dimension posterior PDFs in the parameter space via a sampling procedure based on Markov Chain Monte Carlo (MCMC) theorems (Metropolis et al., 1953; Hastings, 1970). Briefly, the $\mathrm{M}-\mathrm{H}$ algorithm was run by repeating two steps: a proposing step and a moving step (Xu et al., 2006). In the proposing step, a new point $\theta^{\text {new }}$ is generated based on the previously accepted point $\theta^{\text {old }}$ with a proposal distribution $P\left(\theta^{\text {new }} \mid \theta^{\text {old }}\right)$ :

$\theta^{\text {new }}=\theta^{\text {old }}+r\left(\theta_{\max }-\theta_{\min }\right) / D$

where $\theta_{\max }$ and $\theta_{\min }$ are the maximum and minimum values in the prior range of the given 
parameter, $d$ is a random variable between -0.5 and 0.5 with a uniform distribution, and $D$ is used to control the proposing step size and was set to 10 in the current study. In the moving step, the new point $\theta^{\text {new }}$ is tested against the Metropolis criteria (Xu et al., 2006) to examine if it should be accepted or rejected. The $\mathrm{M}-\mathrm{H}$ algorithm was run 500,000 times for each lab incubation data. Accepted parameter values were then used to construct posterior PDFs (Fig.

2).

\section{Statistical analysis}

We found that incubation temperatures significantly affected decomposition rates of the active and the slow $C$ pools (all $P<0.001$, Fig. 3). To exclude the impact of incubation temperatures, we normalized the decomposition rates by using relationships between decomposition rates and incubation temperatures (Fig. 3a-b, equations associated with grey circles and grey lines) before further analyzing the data. Decomposition rates were normalized to a reference temperature of $15^{\circ} \mathrm{C}$ by calculating temperature differences between actual incubation temperatures and $15^{\circ} \mathrm{C}$. We then analyzed the relationships of the normalized decomposition rates with soil properties (including soil sampling depth, bulk density, field water holding capacity, clay content, $\mathrm{C}: \mathrm{N}$ ratio, and $\mathrm{pH}$ values) and environmental factors (including latitude, longitude, mean annual temperature, and mean annual precipitation). Modelled decomposition rates of the passive $C$ pools without normalization were used in analyses because they were not significantly influenced by incubation temperatures (Fig. 3c). We conducted stepwise multiple regression analysis to explore the relationships between decomposition rates and the variables mentioned above (SPSS 17.0 for windows, SPSS Inc., 
Chicago, IL, USA). Additionally, structural equation modeling (SEM) was applied to quantify direct and indirect impacts of environmental variables and soil properties on decomposition rates of SOC pools. Data were fitted to the model using the maximum likelihood estimation method. Adequate model fit was indicated by a non-significant $\chi^{2}$ test $(P>0.05)$. SEM analysis was performed using AMOS 21.0 software (IBM, SPSS, Armonk, NY, USA).

\section{Results}

Decomposition rates of the active and the slow $\mathrm{C}$ pools were mostly constrained, but not for the passive $\mathrm{C}$ pool (Fig. 2). This is because in the measured $\mathrm{CO}_{2}$, only a very small proportion came from the decomposition of the passive $\mathrm{C}$ pool, making it difficult to constrain the parameter with a weak signal. In general, decomposition rates of different SOC pools varied greatly, exhibiting normal distributions with a mean turnover time of $0.2,5$, and $1369 \mathrm{yr}$ for the active, the slow, and the passive SOC pools, respectively (Fig. 4a-c). The strong relationship between the observed and the modeled $\mathrm{CO}_{2}$ fluxes $\left(r^{2}\right)$ indicate that the model performed well (Figs. 2d and 4d).

Linear regression analyses showed that decomposition of the active and the slow organic $C$ pools in soils was regulated by soil physical and chemical properties (all $P<0.05$ ). High clay content (Fig. 5a-b), field water holding capacity (WHC, Fig. 6a-b), and C:N ratio (Fig. 7a-b) all reduced decomposition rates. On the other hand, decomposition of the passive $\mathrm{C}$ pool was not significantly affected by either the environmental factors or soil properties mentioned above (all $P>0.05$, Figs. 5c, 6c, and 7c) except its negative relationship with binned $\mathrm{C}: \mathrm{N}$ ratio $(P<0.05$, 
Fig. 7c). Multiple regressions showed clay and WHC were important in regulating decomposition of the active SOC pool and clay and C:N ratio governed decomposition of the slow SOC pool (Table 1). Soil pH values did not significantly impact SOC decomposition of different pools (all $P>0.05$ ).

Final SEM adequately fitted the data of the interactive networks of environmental factors and soil properties in regulating decomposition of the active $\left(\chi^{2}=11.12, d f=6, P=0.10\right.$, Fig. 8 a) and the slow $\left(\chi^{2}=11.35, \mathrm{df}=6, P=0.10\right.$, Fig. 8 b) SOC pools. The models explained $38 \%$ and $40 \%$ variation in decomposition of the active and the slow pools, respectively. Clay content directly influenced SOC decomposition. Multifactor regression and SEM analyses showed that clay was the most important variable in controlling decomposition of SOC (Table 1, Fig. 8).

\section{Discussion}

Various soil physical and chemical properties (e.g. clay content and C:N ratio) have been reported to govern organic matter decomposition rates by previous studies (e.g. Enríquez et al., 1993; Balesdent et al., 2000; Mtambanengwe et al., 2004; Schädel et al., 2013). However, few studies have examined the intrinsic decay rates of the active, the slow, and the passive SOC pools and their controlling factors at a large spatial scale given the difficulties of directly estimating pool-specific decomposition rates. Results of our data assimilation demonstrated that $\mathrm{C}$ decomposition was negatively regulated by soil properties, including clay content, WHC, and C:N ratio. The inverse relationships are discussed below in detail. Clay - C decomposition relationship 
Soil texture may control SOC decomposition, and its content may give an idea of the degree of protection conferred by a particular soil (Krull et al., 2001; Six et al., 2002). In general, clay content is assumed to be positively correlated with preservation of SOC (Schimel et al., 1985; Spain, 1990; Giardina et al., 2001). In line with many previous empirical studies (e.g. Schimel et al., 1994; Balesdent et al., 2000; Mtambanengwe et al., 2004), we found that clay content negatively regulated decomposition rates of the SOC pools (all $P<0.01$, Fig.5a-b, Table 1). For example, some studies have shown that increasing clay content generally results in higher organic C $(\mathrm{OC})$ in the occluded particulate organic matter (POM, active pool, Kölbl \& Kögel-Knabner, 2004). High soil clay content promotes the conservation of POM with a low degree of alteration (Kölbl \& Kögel-Knabner, 2004). Two mechanisms, the formation of complexes between metal ions associated with large clay surface area and substrate accessibility by microbes, (Krull et al., 2001; Mtambanengwe et al., 2004), could explain the reduced decomposition rates with increasing clay content.

First, size and surface area of clay mineral particles matter. In terms of soil texture, clay particles are associated with small diameters and thus larger surface areas. A decline in SOC decomposition rates with decreasing particle size has been commonly observed (Giardina et al., 2001). In addition, clay, with a permanent negative surface charge, has an advantage of attracting precipitated amorphous Fe and Al oxides on its large surface and then, absorbing negative charged organic matter through cation bridging (Sollins et al., 1996; Krull et al., 2001). Second, pore size distribution matters. Microbes are assumed to mainly live in pores of a certain size (Mtambanengwe et al., 2004). Pore size distribution of a soil thus determines the 
accessibility of organic matter to decomposer organisms (Krull et al., 2001). With increasing clay content, the relative contribution of small pores to the total porosity increases, and decomposition of SOC decreases due to the exclusion of decomposer organisms increases (Mtambanengwe et al., 2004). Our results provide direct evidence to support the theory that decomposition of SOM is controlled by soil texture at a large spatial scale.

On the other hand, the importance of clay content on SOC decomposition could vary across soils with different clay mineralogy (quantity vs. quality) (Spain, 1990; Percival et al., 2000). The physical and chemical properties of a particular clay mineral depend on its structure and composition (Saggar et al., 1996; Schønning et al., 1999). Structurally, arrangement of silicon-oxygen sheet and aluminum-oxygen sheet in clays affect soil porosity (space available for water and air) and therefore, decomposition of SOC. On the clay composition, studies have shown relatively weak correlations between SOC and clay content because of the influence of sesquioxides (Saiz et al., 2012) and pyrophosphate-extractable Al and allophane (Percival et al., 2000). In support of our results, however, SOC in general increases linearly with clay content at regional and global scales due to the role of clay in retention of organic matter (Parton et al., 1993; Schimel et al., 1994; Oades, 1998).

\section{WHC - C decomposition relationship}

The role WHC played in influencing SOC decomposition is dependent on soil texture. Soil texture impacts the physical characteristics of soil, such as WHC (Schønning et al., 1999; Giardina et al., 2001; Krull et al., 2001). The higher the percentage of clay sized particles, the higher the WHC. Clay has a much larger surface area, which allows soils to hold a greater 
quantity of water. In accordance with the study by Schønning et al. (1999), our results showed that decomposition rates of the active and the slow SOC pools significantly decreased with increasing WHC (all $P<0.05$, Fig. 6). WHC governs microbial decomposition of SOC through its influences on soil water availability and the aeration status. With increasing WHC/clay content, soils hold much more water, resulting in less oxygen supply and thus hinder processes of decomposer organisms. The results reported here thereby suggest that decomposition of SOC is regulated by WHC as determined by soil texture.

\section{C:N-C decomposition relationship}

Another aspect of soil properties that affects the rate of SOC decomposition is $\mathrm{C}: \mathrm{N}$ ratio that directly impacts decomposer organisms (Enríquez et al., 1993). As the C:N ratio increased, the decomposition rates of the active and the slow SOC pools greatly decreased (all $P<0.05$, Fig. 7). $\mathrm{N}$ is of essential importance for microbial growth and therefore the breakdown of organic mater. Decay of organic matter can be delayed if $\mathrm{N}$ availability (high $\mathrm{C}: \mathrm{N}$ ratio) in soils is low. Although WHC and C:N ratio entered into model 2 in regulating decomposition of the active and the slow SOC pools, respectively, SEM analysis showed that their impacts on SOC decomposition was much less in comparison to clay content (Table 1, Fig. 8). Our results generally suggest that clay content in soils is probably the most important factor that directly governs SOC stabilization against biological attack.

In addition, our results also showed that decomposition of the active SOC pools decreased with increasing latitude, while increasing with increasing mean annual temperature and precipitation (all $P<0.05$, Fig. 9), likely resulted from geographical differences in temperature 
and precipitation. Generally, low-latitude soils are associated with high annual temperature and precipitation. As a result, decomposition of SOC was greatest in tropical regions. On the other hand, decomposition of the passive SOC pool was not affected by either soil properties or environmental factors (Figs. 5c, 6c, and 7c). Although possible, this also might be because the incubation data did not provide enough information to allow modeling of decay rates of the passive $\mathrm{SOC}$ pool (Fig. 2c). $\mathrm{CO}_{2}$ efflux was dominated by the active pool at the beginning and then by the slow pool during the incubation (Wang et al., 2013).

The limitation of this study is the scarcity of tropical-based studies. There are no tropical grasslands or savannas included in the dataset (Fig. 1). A two-peak distribution histogram both for the active and the slow pools may also result from the scarcity of incubation studies in tropics (Fig. 4a-b), where the turnover rate of SOC is usually high (Wick \& Tiessen, 2008). Tropical forests, grasslands, and savannas are quite complex system, generally occurring on heavily weathered soils. Moreover, grassland-dominated tropical environments are often subjected to recurrent disturbances, such as fires, which may impact the passive and even the slow carbon pool. The pattern of soil properties in controlling SOC decomposition discussed above may thus change to some extent if tropical systems were included.

To conclude, this present study could improve our understanding of decomposition of SOC. To our knowledge, it is unique because we show inverse soil property- $\mathrm{C}$ decomposition relationships at a large spatial scale and moreover, we quantitatively evaluate the essential role of soil texture (clay content) in controlling decomposition of SOC. Our study indicates a general pattern of SOC decomposition at large scales, which not only extends the results of 
small-scale studies but also is very useful for model development and projection of changes in terrestrial $\mathrm{C}$ sequestration in future.

\section{Acknowledgements}

We thank three anonymous referees for their thoughtful comments and suggestions on an earlier draft. We also thank Michelle L. Haddix for providing us incubation data and Jianyang Xia and Nathaniel Mikle for generating the spatial distribution map of individual studies. This study is financially supported by National Science Foundation (NSF) under grant DEB 0743778, the Office of Science (BER), Department of Energy, under grant DE-SC0004601, Jiangsu Specially-Appointed Professors Program, and the Priority Academic Program Development of Jiangsu Higher Education Institutions.

\section{References:}

Baldock, J.A., Skjemstad, J.O., 2000. Role of the soil matrix and minerals in protecting natural organic materials against biological attack. Organic Geochemistry 31, 697-710.

Balesdent, J., Chenu, C., Balabane, M., 2000. Relationship of soil organic matter dynamics to physical protection and tillage. Soil and Tillage Research 53, 215-230.

Burke, I.C., Kaye, J.P., Bird, S.P., Hall, S.A., McCulley, R.L., Sommerville, G.L., 2003. Evaluating and Testing Models of Terrestrial Biogeochemistry: The Role of Temperature in Controlling Decomposition. In Models in Ecosystem Science. Eds. CD Canham, JJ Cole, WK Lauenroth. pp. 225-253. Princeton University Press, 
Princeton, NJ, USA.

Conant, R.T., Drijber, R.A., Haddix, M.L., Parton, W.J., Paul, E.A., Plante, A.F., Six, J., Steinweg, J.M., 2008.

Sensitivity of organic matter decomposition to warming varies with its quality. Global Change Biology 14, 868-877.

Craine, J.M., Fierer, N. \& McLauchlan, K.K., 2010. Widespread coupling between the rate and temperature sensitivity of organic matter decay. Nature Geoscience 3, 854-857.

Cusack, D.F., Torn, M.S., McDowell, W.H. \& Silver, W.L., 2010. The response of heterotrophic activity and carbon cycling to nitrogen additions and warming in two tropical soils. Global Change Biology 16, 2555-2572.

Dutta, K., Schuur, E.A.G., Neff, J.C. \& Zimov, S.A., 2006. Potential carbon release from permafrost soils of Northeastern Siberia. Global Change Biology 12, 1-16.

Elberling, B., Michelsen, A., Schade,I C., Schuur, E.A.G., Christiansen, H.H., Berg, L., Tamstorf, M.P., S Sigsgaard, C., 2013. Long-term $\mathrm{CO}_{2}$ production following permafrost thaw. Nature Climate Change 3, doi: 10.1038/NCLIMATE1995.

Enríquez, S., Duarte, C.M. \& Sand-Jensen, K., 1993. Patterns in decomposition rates among photosynthetic organisms: the importance of detritus C:N:P content. Oecologia 94, 457-471.

Friedlingstein, P., Meinshausen, M., Arora, V.K., Jones, C.D., Anav, A., Liddicoat, S.K. \& Knutti, R., 2014. Uncertainties in CMIP5 climate projections due to carbon cycle feedbacks. Journal of Climate 27, 511-526.

Giardina, C.P., Ryan, M.G., Hubbard, R.M. \& Binkley, D., 2001. Tree species and soil textural controls on carbon and nitrogen mineralization rates. Soil Science Society of America Journal 65, 1272-1279. 
Haddix, M.L., Plante, A.F., Conant, R.T., Six, J., Steinweg, J.M., Magrini-Bair, K., Drijber, R.A., Morris, S.J., Paul, E.A., 2011. The role of soil characteristics on temperature sensitivity of soil organic matter. Soil Science Society of America Journal 75, 56-68.

Hastings, W.K., 1970. Monte-Carlo Sampling Methods Using Markov Chains and Their Applications. Biometrika 57, 97-109.

Kätterer, T., Reichstein, M., Andrén, O. \& Lomander, A., 1998. Temperature dependence of organic matter decomposition: a critical review using literature data analyzed with different models. Biology and Fertility of Soils $27,258-262$.

Knoblauch, C., Beer, C., Sosnin, A., Wagner, D. \& Pfeiffer, E., 2013. Predicting long-term carbon mineralization and trace gas production from thawing permafrost of Northeast Siberia. Global Change Biology 19, 1160-1172.

Kölbl, A., Kögel-Knabner, I., 2004. Content and composition of free and occluded particulate organic matter in a different textured arable Cambisol as revealed by solid-state ${ }^{13} \mathrm{C}$ NMR spectroscopy. Journal of Plant Nutrition and Soil Science 167, 45-53.

Krull, E., Baldock, J. \& Skjemstad, J., 2001. Soil texture effects on decomposition and soil carbon storage. In: Kirchbaum, M.U.F., Mueller, R. (Ed.), Net Ecosystem Exchange Workshop Proceedings, CRC for Greenhouse Accounting, Canberra, Australia, pp. 103-110.

Lavoie, M., Mack, M.C. \& Schuur, E.A.G., 2011. Effects of elevated nitrogen and temperature on carbon and nitrogen dynamics in Alaskan arctic and boreal soils. Journal Geophysical Research 116, G03013, doi:10.1029/2010JG001629.

Lee, H., Schuur, E.A.G., Inglett, K.S., Lavoie, M. \& Chanton, J.P., 2012. The rate of permafrost carbon release 
under aerobic and anaerobic conditions and its potential effects on climate. Global Change Biology 18, 515-527.

Liang, J., Li, D., Shi, Z., Tiedje, J.M., Zhou, J., Schuur, E.A.G., Konstantinidis, K.T., Luo, Y., 2015. Methods for estimating temperature sensitivity of soil organic matter on incubation data: a comparative evaluation. Soil Biology and Biochemistry 80, 127-135.

Luo, Y., Weng, E., 2011. Dynamic disequilibrium of terrestrial carbon cycle under global change. Trends in Ecology and Evolution 26, 96-104.

Luo, Y., White, L.W., Canadell, J.G., DeLucia, E.H., Ellsworth, D.S., Finzi, A., Lichter, J., Schlesinger, W.H.,2003. Sustainability of terrestrial carbon sequestration: A case study in Duke Forest with inversion approach. Global Biogeochemical Cycles 17, 1021, doi: 10.1029/2002GB001923.

McGill, W.B., 1996. Review and classification of ten soil organic matter models. In Evaluation of Soil Organic Matter Models. Eds. DS Powlson, P Smith and JU Smith. pp. 111-132. NATO ANSI Series, Springer Verlag. Metropolis, N.A., Rosenbluth, W., Rosenbluth, M.N., Teller, A.H. \& Teller, E. (1953) Equation of state calculations by fast computing machines. The Journal of Chemical Physics 21, 1087-1092.

Mtambanengwe, F., Mapfumo, P., Kirchma, H., 2004, Decomposition of organic matter in soil as influenced by texture and pore size distribution. In: Bationo, A. (Ed.), Managing Nutrient Cycles to Sustain Soil Fertility in Sub-Saharan Africa. Academy Science Publishers and TSBF CIAT, Nairobi, Kenya, pp. 261-275.

Neff, J.C., Hooper, D.U., 2002. Vegetation and climate controls on potential $\mathrm{CO}_{2}$, DOC, and DON production in northern latitude soils. Global Change Biology 8, 872-884.

Oades, J.M., 1998. The retention of organic matter in soils. Biogeochemistry 5, 35-70.

Parton, W.J., Scurlock, J.M.O., Ojima, D.S., Gilmanov, T.G., Scholes, R.G., Schimel, D.S., Kirchner, T., Menaut, 
J.C., Seastedt, T., 1993. Observations and modeling of biomass and soil organic matter dynamics for the grassland biome worldwide. Global Biogeochemical Cycles 7, 785-809.

Percival, H.J., Parfitt, R.L., Scott, N.A., 2000. Factors controlling soil carbon levels in New Zealand grasslands: is clay content important? Soil Science Society of American Journal 64, 1623-1630.

Rey, A., Jarvis, P., 2006. Modelling the effect of temperature on carbon mineralization rates across a network of European forest sites (FORCAST) Global Change Biology 12, 1894-1908.Rey, A., Pegoraro, E., Jarvis, P.G., 2008. Carbon mineralization rates at different soil depths across a network of European forest sites (FORCAST). European Journal of Soil Science 59, 1049-1062.

Saggar, S., Parshotam, A., Sparling, G.P., Feltham, C.W., Hart, P.B.S., 1996. ${ }^{14}$ C-labelled ryegrass turnover and residence times in soils varying in clay content and mineralogy. Soil Biology and Biochemistry 28, 1677-1686.

Saiz, G., Bird, M.I., Domingues, T., Schrodt, F.A., Schwarz, M., Feldpausch, T.R., Veenendaal, E., Djagbletey, G., Hien, F., Compaore, H., Diallo, A., Lloyd, J., 2012. Variation in soil carbon stocks and their determinants across a precipitation gradient in West Africa. Global Change Biology 18, 1670-1683.

Schaeffer, S.M., Billings, S.A., Evans, D., 2007, Laboratory incubations reveal potential responses of soil nitrogen cycling to changes in soil $\mathrm{C}$ and $\mathrm{N}$ availability in Mojave Desert soils exposed to elevated atmospheric $\mathrm{CO}_{2}$. Global Change Biology 13, 854-865.

Schädel, C., Schuur, E.A.G., Bracho, R., Elberling, B., Knoblauch, C., Lee, H., Luo, Y., Shaver, G.R., Turetsky, M.R., 2013. Circumpolar assessment of permafrost $C$ quality and its vulnerability over time using long-term incubation data. Global Change Biology 20, 641-652.

Schjønning, P., Thomsen, I,K,, Møber, J,P,, de Jong, H., Kristensen, K., Christensen, B.T., 1999. Turnover of 
organic matter in differently textured soils: I. Physical characteristics of structurally disturbed and intact soils. Geoderma 89, 177-198.

Schimel, D.S., Braswell, B.H., Holland, E.A., McKeown, R., Ojima, D.S., Painter, T.H., Parton, W.J. \& Townsend, A.R. (1994) Climatic, edaphic, and biotic controls over storage and turnover of carbon in soils. Global Biogeochemical Cycles 8, 279-293.

Schimel, D.S., Coleman, D.C., Horton, K.A., 1985. Soil organic matter dynamics in paired rangeland and cropland toposequences in North Dakota. Geoderma 36, 201-214.

Six, J., Conant, R.T., Paul, E.A., Paustian, K., 2002. Stabilization mechanisms of soil organic matter: implications for C-saturation of soils. Plant Soil 241, 155-176.

Spain, A.V. 1990. Influence of environmental conditions and some soil chemical properties on the carbon and nitrogen contents of some Australian rainforest soils. Australian Journal of Soil Research 28, 825-839.

Stewart, C.E., Paustian, K., Conant, R.T., Plante, A.F., Six, J., 2009. Soil carbon saturation: implications for measurable carbon pool dynamics in long-term incubation. Soil Biology and Biochemistry 41, 357-366.

Thomsen, I.K., Schjønning, P., Jensen, B., Kirstensen, K., Christensen, B.T., 1999. Turnover of organic matter in different textured soils. II. Microbial activity as influenced by soil water regimes. Geoderma 89, 199-218.

Trumbore, S.E., 1997. Potential responses of soil organic carbon to global environmental change. Proceedings of the National Academy of Sciences of the United States of America 94, 8284-8291.

Wang, G., Zhou, Y., Xu, X., Ruan, H., Wang, J., 2013. Temperature Sensitivity of Soil Organic Carbon Mineralization along an Elevation Gradient in the Wuyi Mountains, China. PLoS ONE 8(1): e53914. doi:10.1371/journal.pone.0053914.

Wick, B., Tiessen, H., 2008. Organic matter turnover in light fraction and whole soil under silvopastoral land use in 
semiarid northeast Brazil. Rangeland Ecology and Management 61(3), 275-283.

Xu, T., White, L., Hui, D., Luo, Y., 2006. Probability inversion of a terrestrial ecosystem model: analysis of uncertainty in parameter estimation and model prediction. Global Biogeochemical Cycles 20, GB2007, doi:10.1029/2005GB002468.

Xu, X., Cheng, X., Zhou, Y., Luo, Y., Ruan, H., Wang, J., 2010a. Variation of soil organic carbon pools along an elevational gradient in the Wuyi Mountains, China. Journal of Resources and Ecology 1, 368-374.

Xu, X., Zhou, Y., Ruan, H., Luo, Y., Wang, J., 2010b. Temperature sensitivity increases with soil organic carbon recalcitrance along an elevational gradient in the Wuyi Mountains, China. Soil Biology and Biochemistry $42,1811-1815$. 
Table 1 Results of multi-regression analysis of decomposition of SOC with soil properties and environmental factors.

\begin{tabular}{|c|c|c|c|c|c|c|}
\hline & Model & Variable & Regression & $\mathrm{n}$ & $r^{2}$ & $P$ \\
\hline \multirow{2}{*}{$\mathrm{k}_{1}$} & 1 & Clay & $\mathrm{k}_{1}=-0.44$ clay +26.83 & 75 & 0.25 & $<0.001$ \\
\hline & 2 & Clay, WHC & $\mathrm{k}_{1}=-0.58$ clay $-9.44 \mathrm{WHC}+35.61$ & 75 & 0.34 & $<0.001$ \\
\hline \multirow{2}{*}{$\mathrm{k}_{2}$} & 1 & Clay & $\mathrm{k}_{2}=-0.02$ clay +0.92 & 75 & 0.25 & $<0.001$ \\
\hline & 2 & Clay, C:N & $\mathrm{K}_{2}=-0.02$ clay $-0.02 \mathrm{C}: \mathrm{N}+1.24$ & 75 & 0.35 & $<0.001$ \\
\hline
\end{tabular}

Note: $k_{1}$ and $k_{2}$, decomposition rates of the active and the slow C pools, respectively; WHC, water holding capacity. Variables used in the regression analysis: latitude, mean annual temperature, mean annual precipitation, WHC, C:N ratio and clay content. 


\section{Figure legends}

Figure 1 Locations of individual studies included in this synthesis $(n=73)$. Some individual studies contain multiple sampling sites.

Figure 2 An example of frequency distribution of the posterior PDFs of specific decomposition rates of the active (a), slow (b), and passive (c) soil organic carbon pools. Inserted panel d shows the relationship between observed and modelled cumulative $\mathrm{CO}_{2}$ fluxes in $\mathrm{mg} \mathrm{C} \mathrm{g}{ }^{-1}$ soil. Observed $\mathrm{CO}_{2}$ data is obtained from Rey et al. (2008) Hardwood site, soil layer of 10-20 cm. A maximum likelihood estimate (MLE) was calculated for each of the well-constrained parameters (a-b), while a mean value was calculated for the poorly constrained parameter (c).

Figure 3 Relationships of incubation temperatures with decomposition rates of the active (a), slow (b), and passive (c) SOC pools. Grey circles and solid grey lines in the panels represent the decomposition rates and their relationships with incubation temperatures $(n=376)$. Circles and lines in color represent the binned decomposition rates and their relationships with incubation temperatures at $4,5,10,15,20,25,30$, and $35^{\circ} \mathrm{C}(\mathrm{n}=8)$, respectively. Equations are associated with the grey lines.

Figure 4 Frequency distribution of decomposition rates of the active (a), the slow (b), and the passive (c) SOC pools and the $r$ square values between observed and modelled cumulative $\mathrm{CO}_{2}$ fluxes for the whole data sets $(\mathrm{n}=376)$. The solid curves are Gaussian distributions fitted to the frequency data.Figure $\mathbf{5}$ Relationships of clay with decomposition rates of the active (a), the slow (b), and the passive (c) SOC pools ( $n=197)$. Grey circles, which are 
associated by solid grey lines, represent the normalized decomposition rates. Circles in color, which are associated by color lines, represent the binned decomposition rates at a $2.5 \%$ clay content interval (points when $\mathrm{n}<3$ were excluded). The grey and the color lines do not statistically differ from each other in each panel (all $P>0.05$ ). Equations are associated with the grey lines.

Figure 6 Relationships of water holding capacity (WHC) with decomposition rates of the active (a), the slow (b), and the passive (c) SOC pools ( $n=197)$. Grey circles, which are associated by solid grey lines, represent the normalized decomposition rates. Circles in color, which are associated by color lines, represent the binned decomposition rates at a $0.1 \mathrm{WHC}$ interval. The grey and the color lines do not statistically differ from each other in each panel (all $P>0.05$ ). Equations are associated with the grey lines.

Figure 7Relationships of $\mathrm{C}: \mathrm{N}$ ratio with decomposition rates of the active (a), the slow (b), and the passive (c) SOC pools ( $n=359)$. Grey circles, which are associated by solid grey lines, represent the normalized decomposition rates. Circles in color, which are associated by color lines, represent the binned decomposition rates at a $2 \mathrm{C}: \mathrm{N}$ ratio interval. The grey and the color lines do not statistically differ from each other in each panel (all $P>0.05$ ). Equations are associated with the grey lines.

Figure 8 Path analysis shows direct and indirect effects on decomposition of the active (a) and the slow SOC pools. Solid and dashed arrows represent significant $(P<0.05)$ and non-significant $(P>0.05)$ paths in a fitted structural equation model depicting impact of variables on the decomposition rates. LAT, latitude; MAT and MAP, mean annual 
temperature and precipitation; WHC, water holding capacity.

Figure 9 Relationships of decomposition rates of the active SOC pool with latitude $(a, n=380)$, mean annual temperature (MAT, $b, n=365)$, and mean annual precipitation (MAP, $c$, $\mathrm{n}=338$ ). Grey circles, which are associated by solid grey lines, represent the normalized decomposition rates. Circles in color, which are associated by colorized lines, represent the binned decomposition rates at $5^{\circ}$ latitude, $5{ }^{\circ} \mathrm{C}$ MAT, and $100 \mathrm{~mm}$ MAP intervals (points when $\mathrm{n}<3$ were excluded. Equations are associated with the grey lines. 


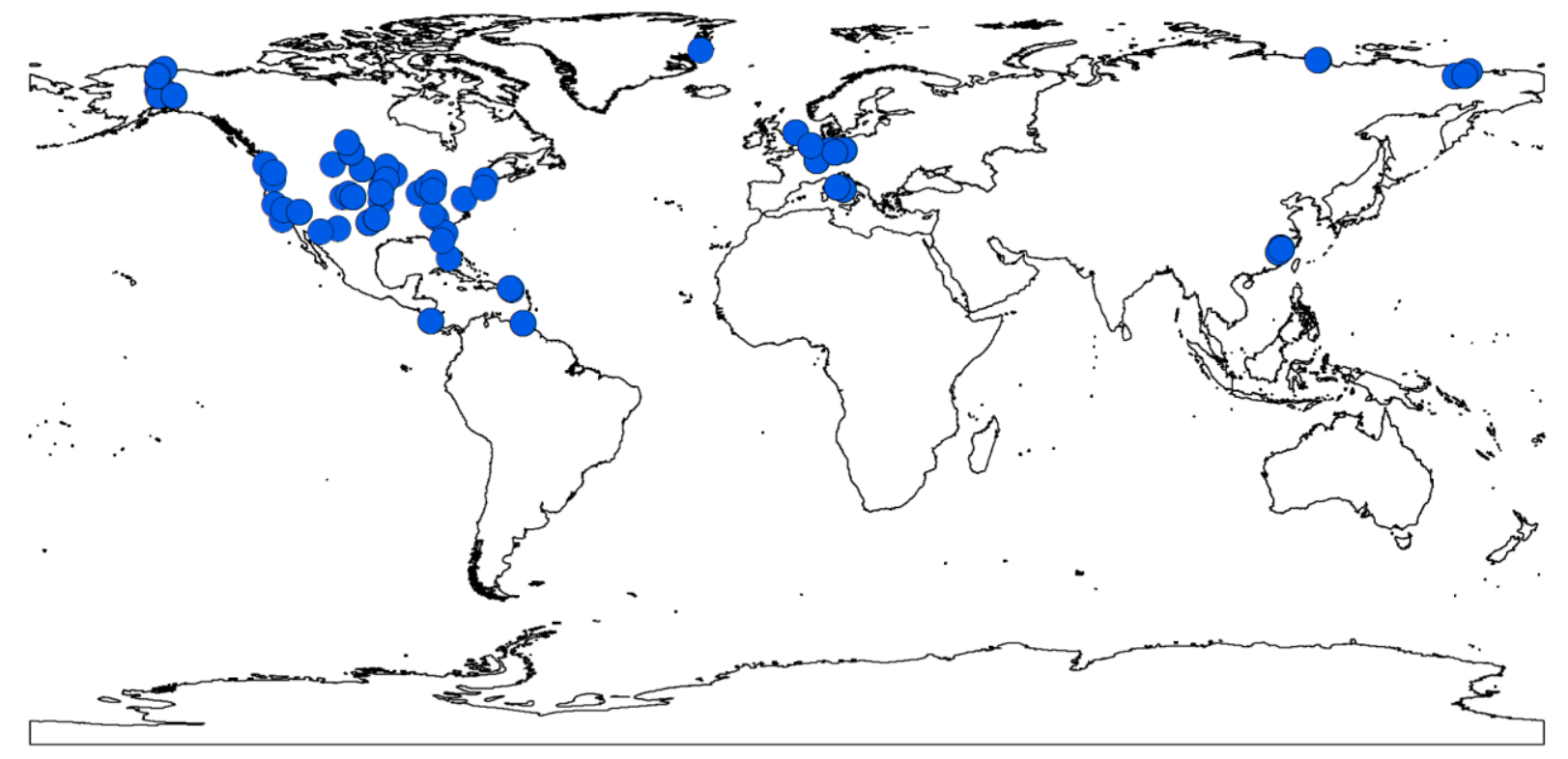

Fig. 1

5 


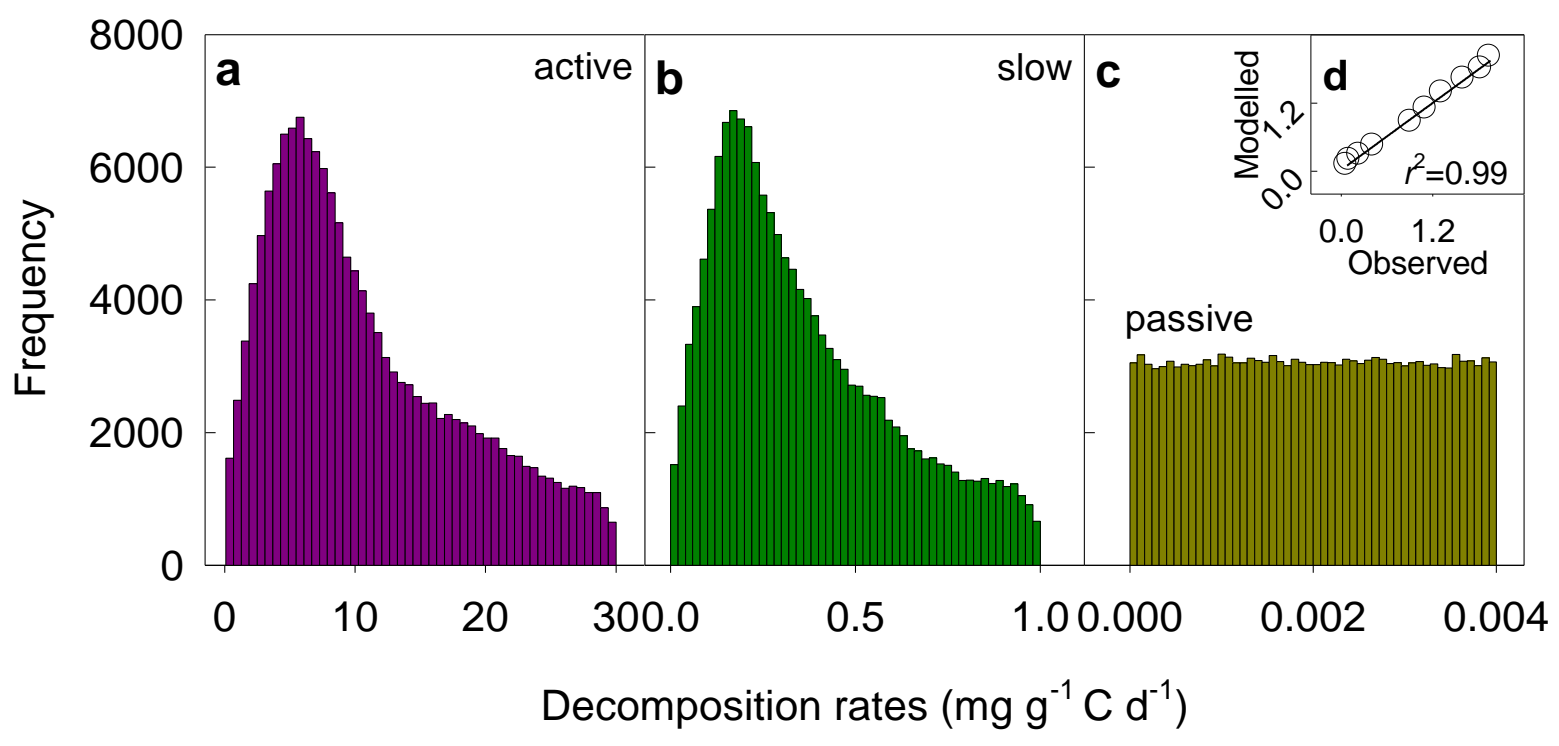

Fig. 2 


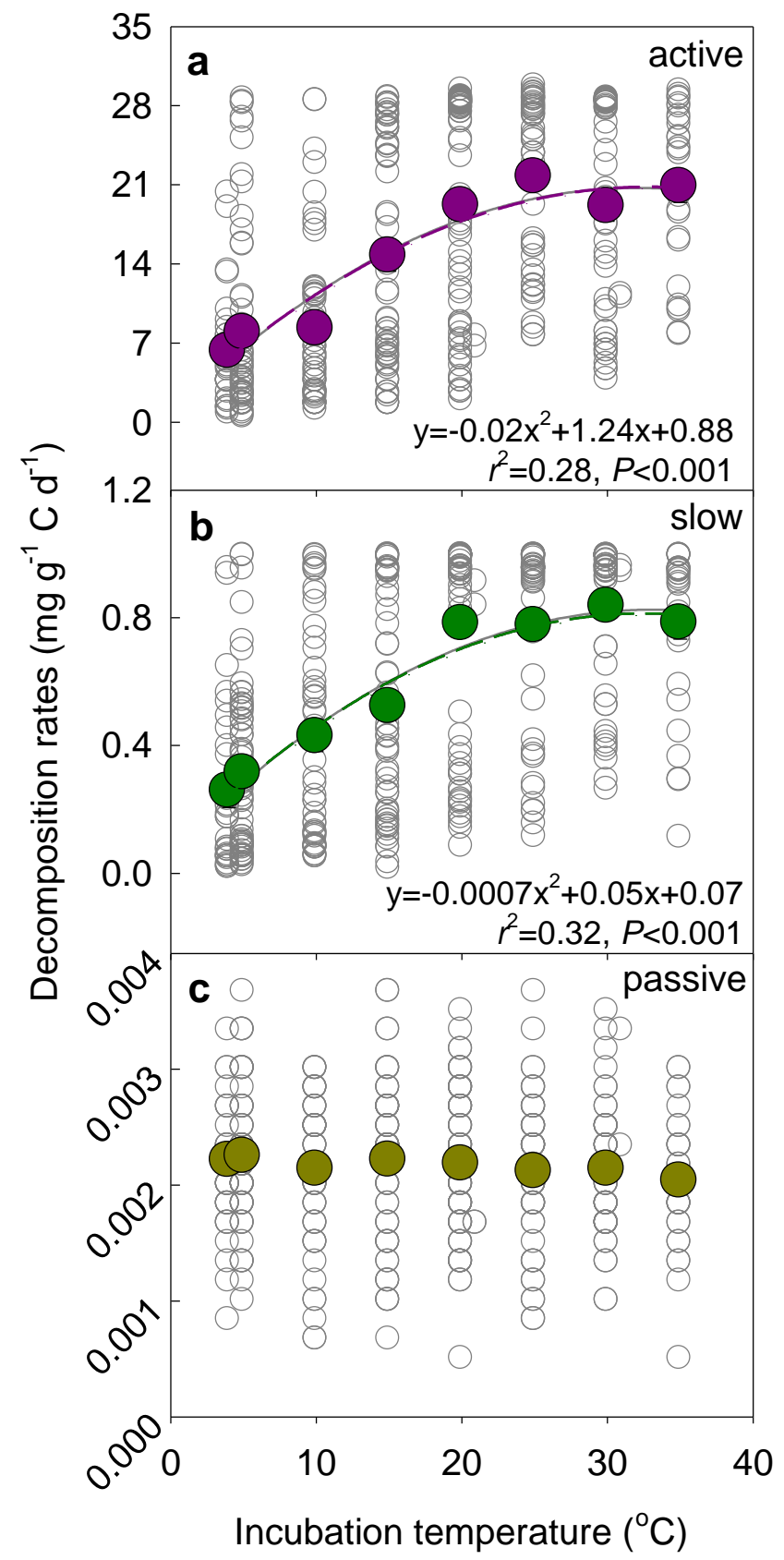

Fig. 3 

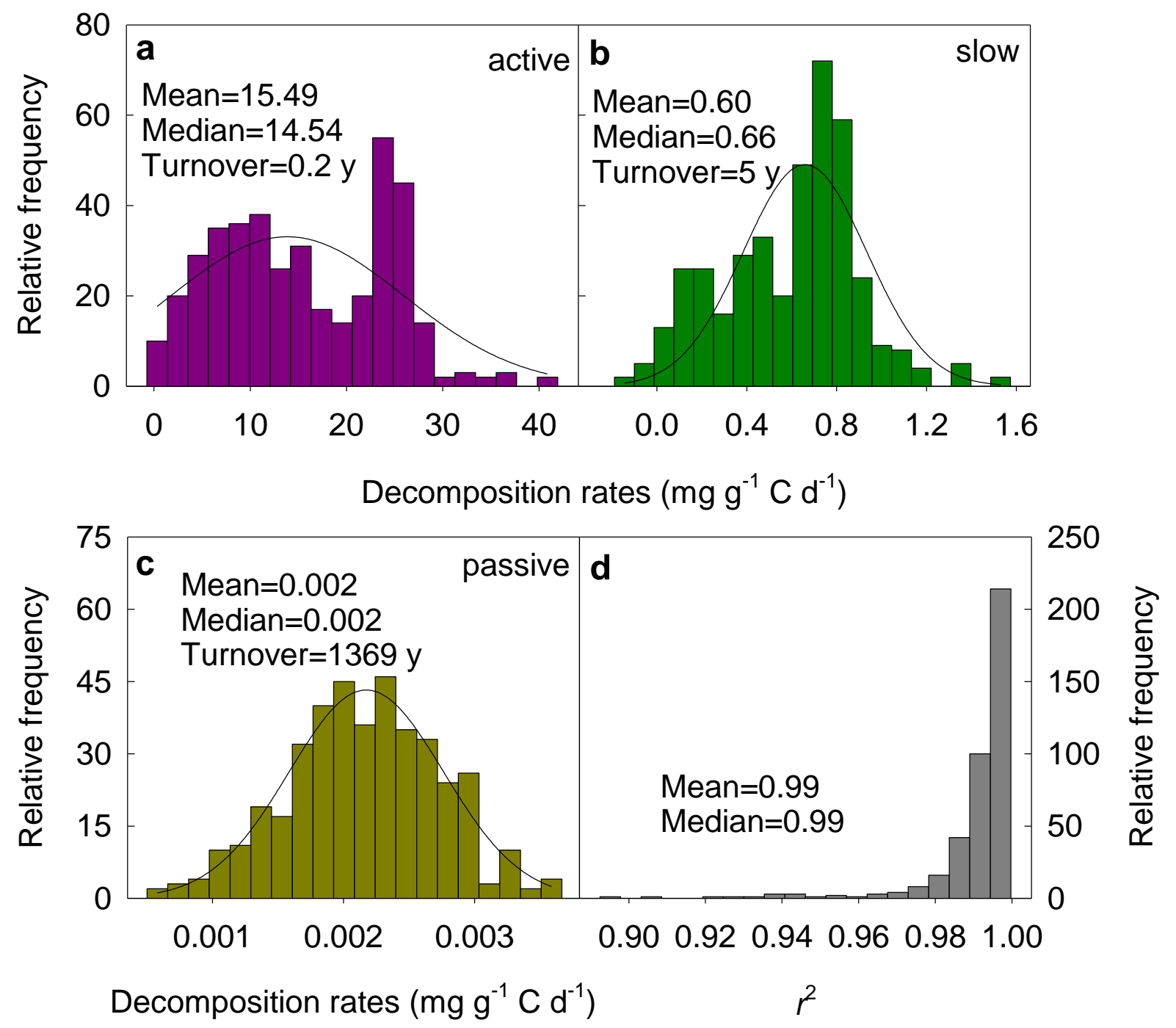

Fig. 4 


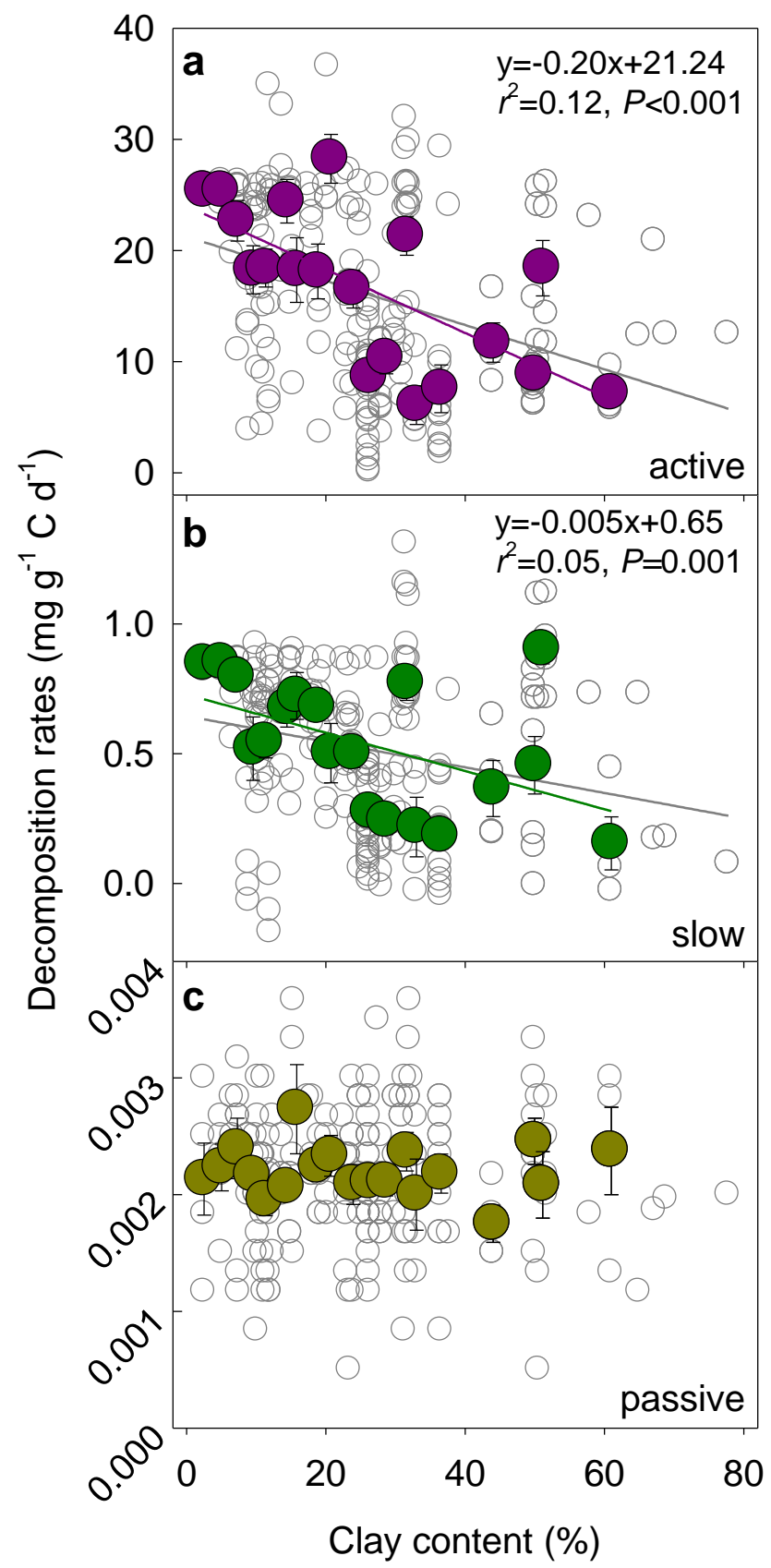

Fig. 5 


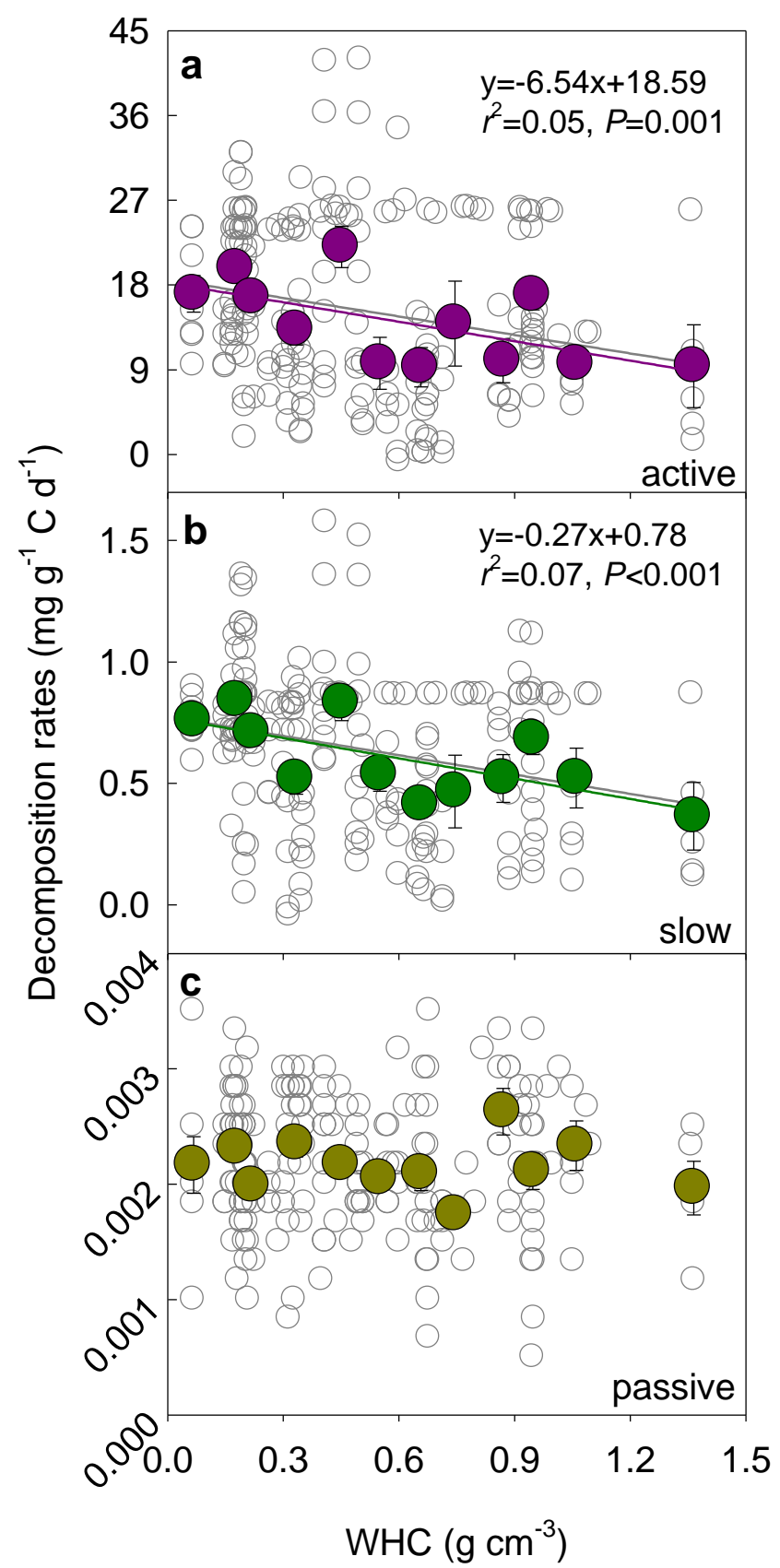

Fig. 6 


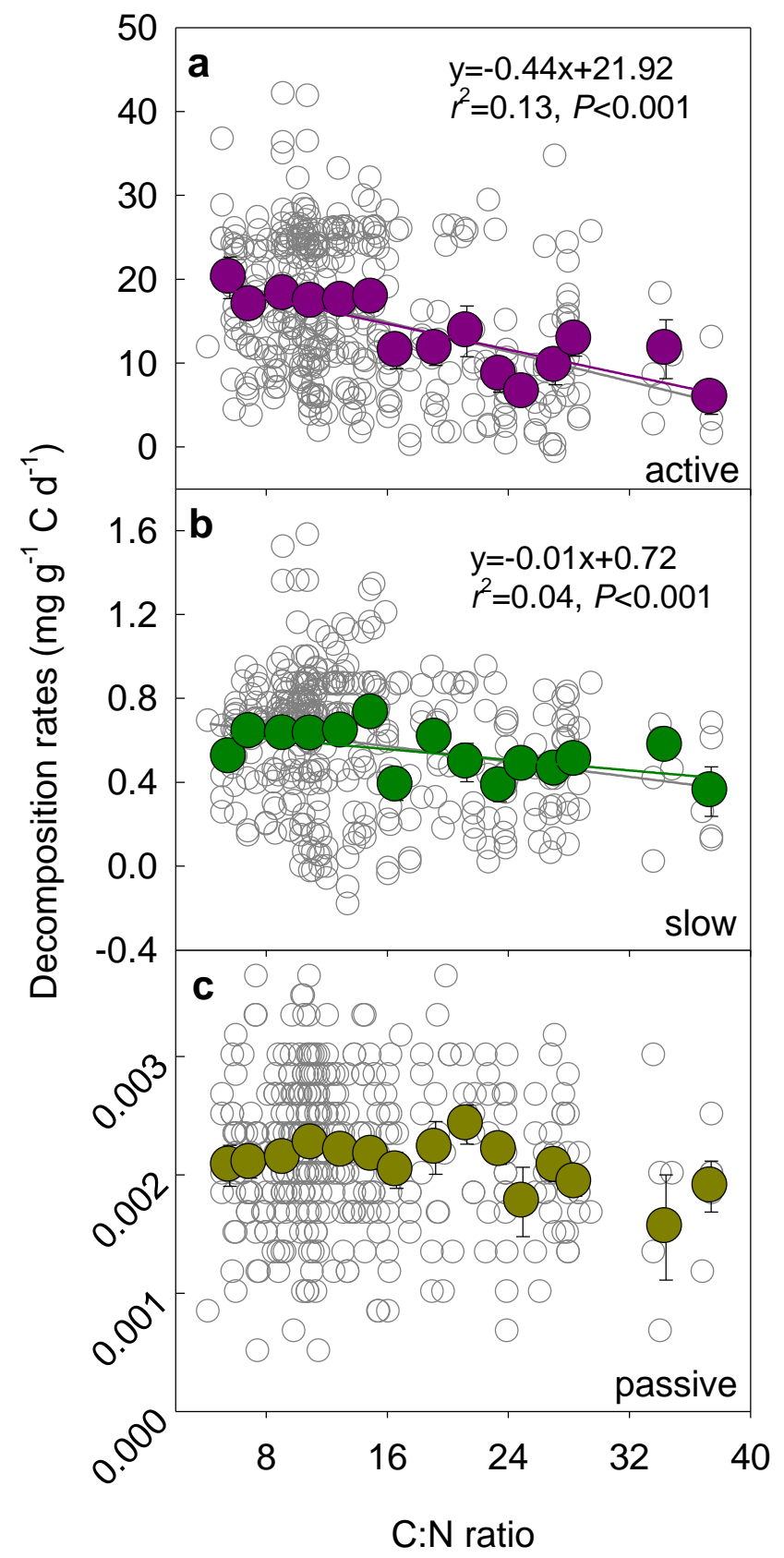

Fig. 7 


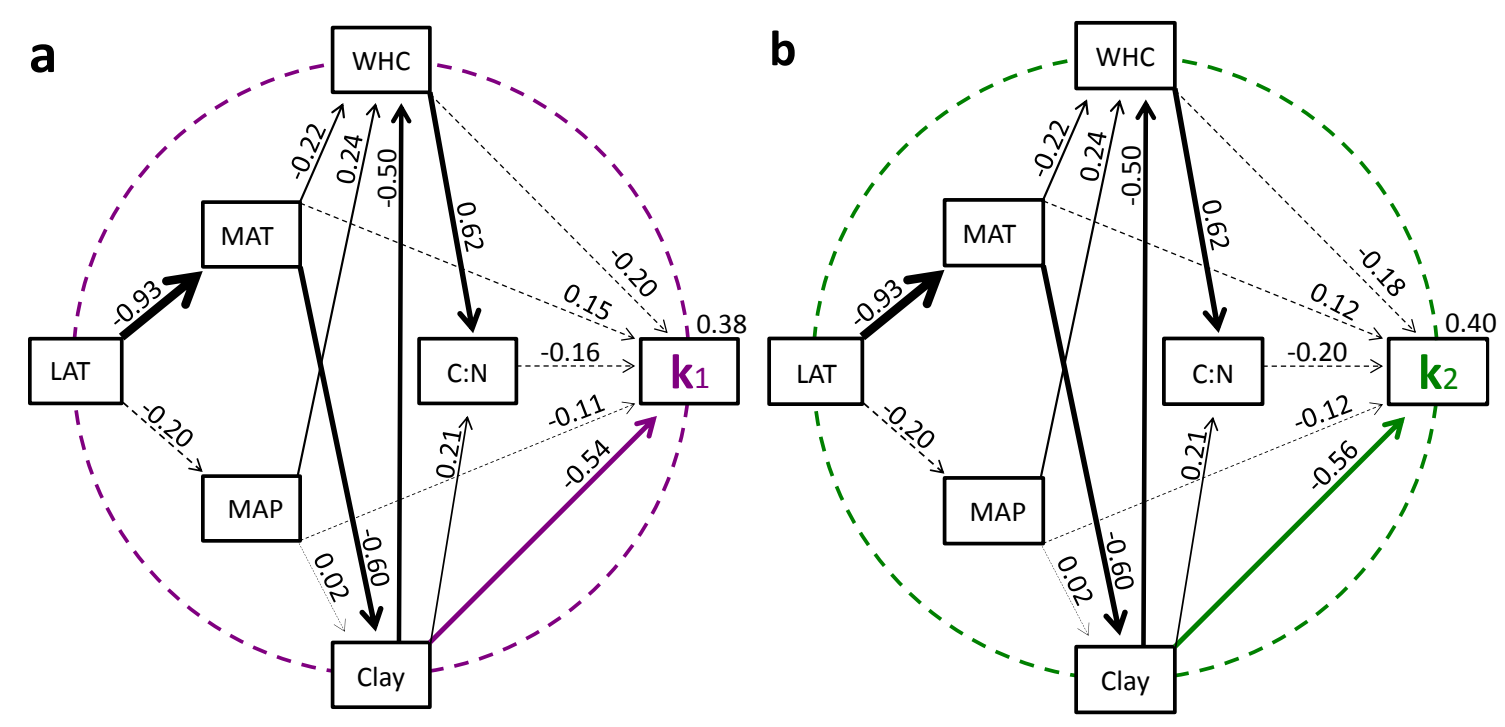

Fig. 8 


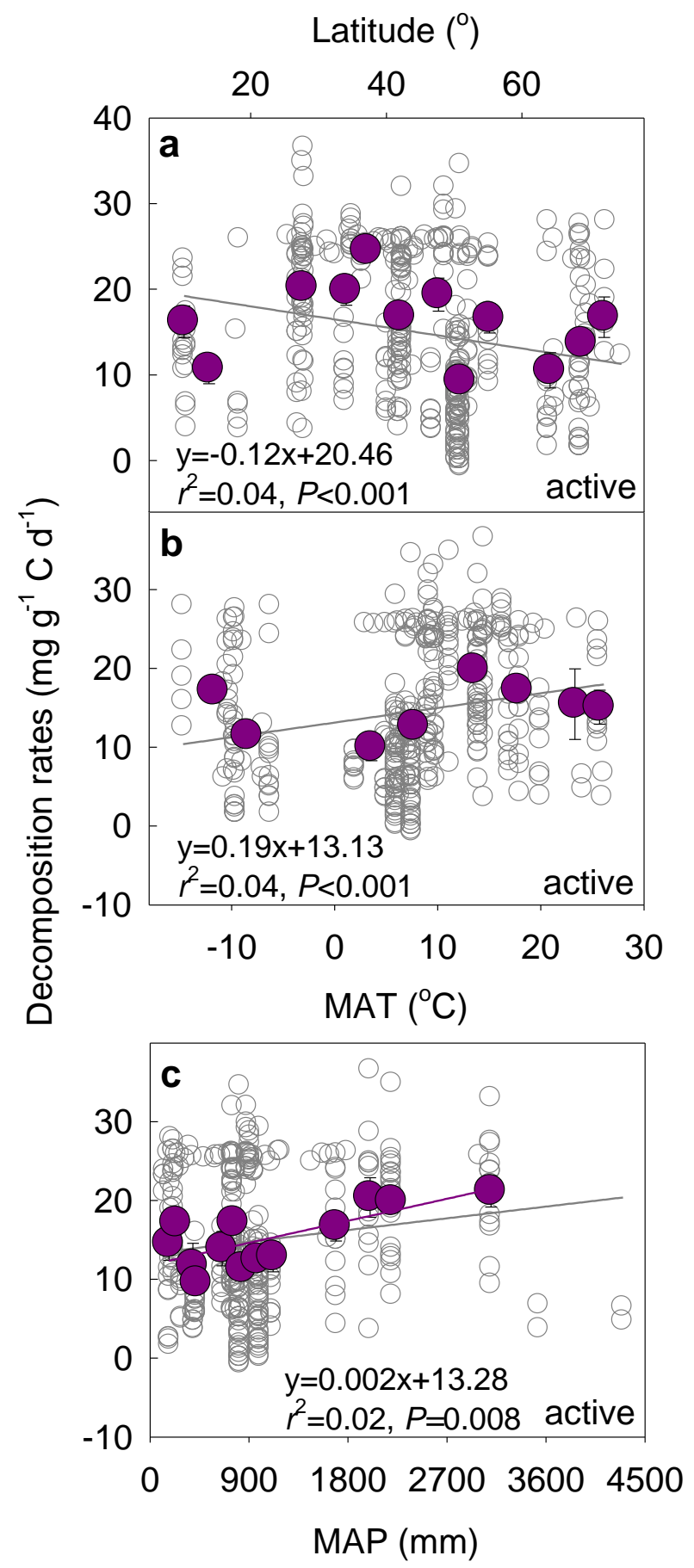

Fig. 9 